\title{
Where does Neisseria acquire foreign DNA from: an examination of the source of genomic and pathogenic islands and the evolution of the Neisseria genus
}

Catherine Putonti ${ }^{1,2,3^{*}}$, Bogdan Nowicki ${ }^{4}$, Michael Shaffer ${ }^{1,3}$, Yuriy Fofanov ${ }^{5,6}$ and Stella Nowicki ${ }^{4}$

\begin{abstract}
Background: Pathogenicity islands (PAIs) or genomic islands (GEls) are considered to be the result of a recent horizontal transfer. Detecting PAIs/GEls as well as their putative source can provide insight into the organism's pathogenicity within its host. Previously we introduced a tool called S-plot which provides a visual representation of the variation in compositional properties across and between genomic sequences. Utilizing S-plot and new functionality developed here, we examined 18 publicly available Neisseria genomes, including strains of both pathogenic and non-pathogenic species, in order to identify regions of unusual compositional properties (RUCPs) using both a sliding window as well as a gene-by-gene approach.
\end{abstract}

Results: Numerous GEls and PAls were identified including virulence genes previously found within the pathogenic Neisseria species. While some genes were conserved amongst all species, only pathogenic species, or an individual species, a number of genes were detected that are unique to an individual strain. While the majority of such genes have an origin unknown, a number of putative sources including pathogenic and capsule-containing bacteria were determined, indicative of gene exchange between Neisseria spp. and other bacteria within their microhabitat. Furthermore, we uncovered evidence that both N. meningitidis and N. gonorrhoeae have separately acquired DNA from their human host. Data suggests that all three Neisseria species have received horizontally transferred elements post-speciation.

Conclusions: Using this approach, we were able to not only find previously identified regions of virulence but also new regions which may be contributing to the virulence of the species. This comparative analysis provides a means for tracing the evolutionary history of the acquisition of foreign DNA within this genus. Looking specifically at the RUCPs present within the 18 genomes considered, a stronger similarity between $N$. meningitidis and $N$. lactamica is observed, suggesting that N. meningitidis arose before N. gonorrhoeae.

Keywords: Neisseria, Horizontal gene transfer, Pathogenicity islands, Pathogen-host DNA transfer

\footnotetext{
* Correspondence: cputonti@luc.edu

'Department of Biology, Loyola University Chicago, 1032 W. Sheridan Rd, Chicago, IL 60660, USA

2Department of Computer Science, Loyola University Chicago, Chicago, IL 60660, USA

Full list of author information is available at the end of the article
} 


\section{Background}

Infectious diseases, which remain a major cause of human morbidity/mortality, are the direct result of interactions between the human host and unique sets of microbial virulence factors. These virulence factors have often been found in clusters of horizontally transferred 10-100 kb genomic DNA regions [1-3] or in short scattered virulence-associated 1-10 kb islets [4]. Regions of DNA introduced through horizontal transfer, referred to as genomic islands (GEIs), can encode for genes of a variety of functions. Identification of the subset of GEIs that encode for virulence factors, referred to as pathogenicity islands (PAIs), can lead to the understanding of the organism's pathogenicity within the host.

There is considerable interest in developing statistical methods to predict PAIs and/or GEIs for future experimental validation as well as furthering our understanding of pathogenicity. As such, numerous statistical methods have been developed to identify aberrant regions in terms of nucleotide composition [5-20] as well as examining phylogenetic discrepancies [21-27]. While alone each of these techniques is appropriate for the detection of a particular GEI/PAI feature, no single approach is capable of detecting all GEIs and PAIs. Combinatorial approaches, e.g. [28-33], although often more cumbersome and inconclusive (in the case where a region is identified by just some of the measures used), can discover more GEIs and PAIs. Beyond just detecting GEIs and PAIs is the task of identifying the source of the genes acquired. This is further complicated when looking at species with open pan-genomes (in which new strains always include novel genes) such as Neisseria meningitidis [34-36].

A graphic approach called Similarity Plot or S-plot for rapid large scale comparison, analysis, and visualization of genomic sequences was previously employed to recognize regions within a genomic sequence that originated through horizontal gene transfer as well as genes under specific selective forces [37]. This tool, as described in detail in our previous work [37], identifies regions of unusual compositional properties (RUCPs) via a sliding-window approach. Recently we expanded this functionality, permitting gene-by-gene comparative analyses to be performed.

Herein we present the results of a comparative analysis of all complete annotated Neisseria genomes currently available. This includes 18 genomes: three from the species $N$. gonorrhoeae, 14 from the species $N$. meningitidis and one from the non-pathogenic species $N$. lactamica. As previous analysis has shown, $N$. meningitidis exhibits a moderately strong codon bias effect $[38,39]$. As such, recently acquired genes will likely exhibit a codon usage more attuned to the tRNA abundances within their native genome. Through the identification of horizontally acquired elements, we can better understand the evolution of this genus with respect to the transformations occurring at the genomic level as well as the variations in virulence between the species and strains. Looking specifically at the RUCPs present within all 18 genomes considered, a stronger similarity between $N$. meningitidis and $N$. lactamica is observed, suggesting that $N$. meningitidis arose before $N$. gonorrhoeae as shown in previous studies [40].

\section{Results}

Visualizing similarities and dissimilarities in compositional properties within Neisseria spp

For each of the 18 Neisseria strains listed in Table 1, the genomic sequence was compared to itself based upon its frequency distribution of 6-mers within a sliding window of 5,000 nucleotides (Figure 1). (See Methods for discussion regarding the selection of the subsequence size selection.) By comparing a genome to itself, as shown in Figure 1, it is possible to identify windows with a composition varying from the genomic norm (indicated as the blue lines traversing the S-plot) as well as their distribution throughout the genome. Comparisons between different species and strains revealed rearrangements as well as regions present in one genome and absent from another (Additional file 1: Figure S1, Additional file 2: Figure S2, Additional file 3: Figure S3 and Additional file 4: Figure S4). Additional file 5: Figure S5 compares the $N$. meningitidis serogroup C strain FAM18 with (a) an $N$. meningitidis serogroup A strain, (b) an $N$. gonorrhoeae strain, and (c) the $N$. lactamica strain, highlighting the location of genes associated with the capsule; while the $N$. meningitidis strains contain the entire capsule-synthesis (cps) gene cluster, $N$. gonorrhoeae and $N$. lactamica genomes do not $[41,42]$.

\section{Recognizing RUCPs}

Examination of the 18 Neisseria genomes was conducted using both a window-by-window approach, with a window size of 5000 nucleotides, as well as a gene-by-gene approach. (See Methods for a detailed description of each). We refer to the regions exhibiting unusual compositional properties from the window-by-window analysis as wRUCPs and from the gene-by-gene analysis as gRUCPs. Windows/genes can exhibit unusual compositional properties as a result of horizontal gene transfer or specific selective forces. The number of wRUCPs and gRUCPs varied from strain to strain as well as serogroup to serogroup. In total 414 wRUCPs and 2,172 gRUCPs were identified (Table 1). While the $N$. meningitidis WU 2594 strain (serogroup A) isolated from a patient suffering from acute bacterial meningitidis [43] contained the fewest wRUCPs, 144 genes were 
Table 1 Number of RUCPs identified for each Neisseria strain

\begin{tabular}{|c|c|c|c|c|}
\hline Species/strain & Accession & Serogroup & \# wRUCP & \# gRUCP \\
\hline N. gonorrhoeae FA 1090 & NC_002946 & $\mathrm{Ng}$ & 16 & 124 \\
\hline N. gonorrhoeae NCCP11945 & NC_011035 & $\mathrm{Ng}$ & 27 & 78 \\
\hline N. gonorrhoeae TCDC-NG08107 & NC_017511 & $\mathrm{Ng}$ & 22 & 122 \\
\hline N. meningitidis Z2491 & NC_003116 & $\mathrm{NmA}$ & 21 & 141 \\
\hline N. meningitidis WUE 2594 & NC_017512 & $\mathrm{NmA}$ & 10 & 144 \\
\hline N. meningitidis MC58 & NC_003112 & $\mathrm{NmB}$ & 26 & 62 \\
\hline N. meningitidis alpha710 & NC_017505 & $\mathrm{NmB}$ & 27 & 126 \\
\hline N. meningitidis G2136 & NC_017513 & $\mathrm{NmB}$ & 24 & 146 \\
\hline N. meningitidis M01-240149 & NC_017514 & $\mathrm{NmB}$ & 25 & 135 \\
\hline N. meningitidis M04-240196 & NC_017515 & $\mathrm{NmB}$ & 25 & 103 \\
\hline N. meningitidis $\mathrm{H} 44 / 76$ & NC_017516 & $\mathrm{NmB}$ & 24 & 121 \\
\hline N. meningitidis M01-240355 & NC_017517 & $\mathrm{NmB}$ & 28 & 141 \\
\hline N. meningitidis NZ-05/33 & NC_017518 & $\mathrm{NmB}$ & 24 & 128 \\
\hline N. meningitidis FAM18 & NC_008767 & $\mathrm{NmC}$ & 23 & 131 \\
\hline N. meningitidis 053442 & NC_010120 & $\mathrm{NmC}$ & 18 & 82 \\
\hline N. meningitidis 8013 & NC_017501 & $\mathrm{NmC}$ & 25 & 116 \\
\hline N. meningitidis alpha14 & NC_013016 & $\mathrm{NmCln}$ & 27 & 126 \\
\hline \multirow[t]{2}{*}{ N. lactamica ST-640 } & NC_014752 & $\mathrm{Nl}$ & 22 & 146 \\
\hline & & Total: & 414 & 2172 \\
\hline
\end{tabular}
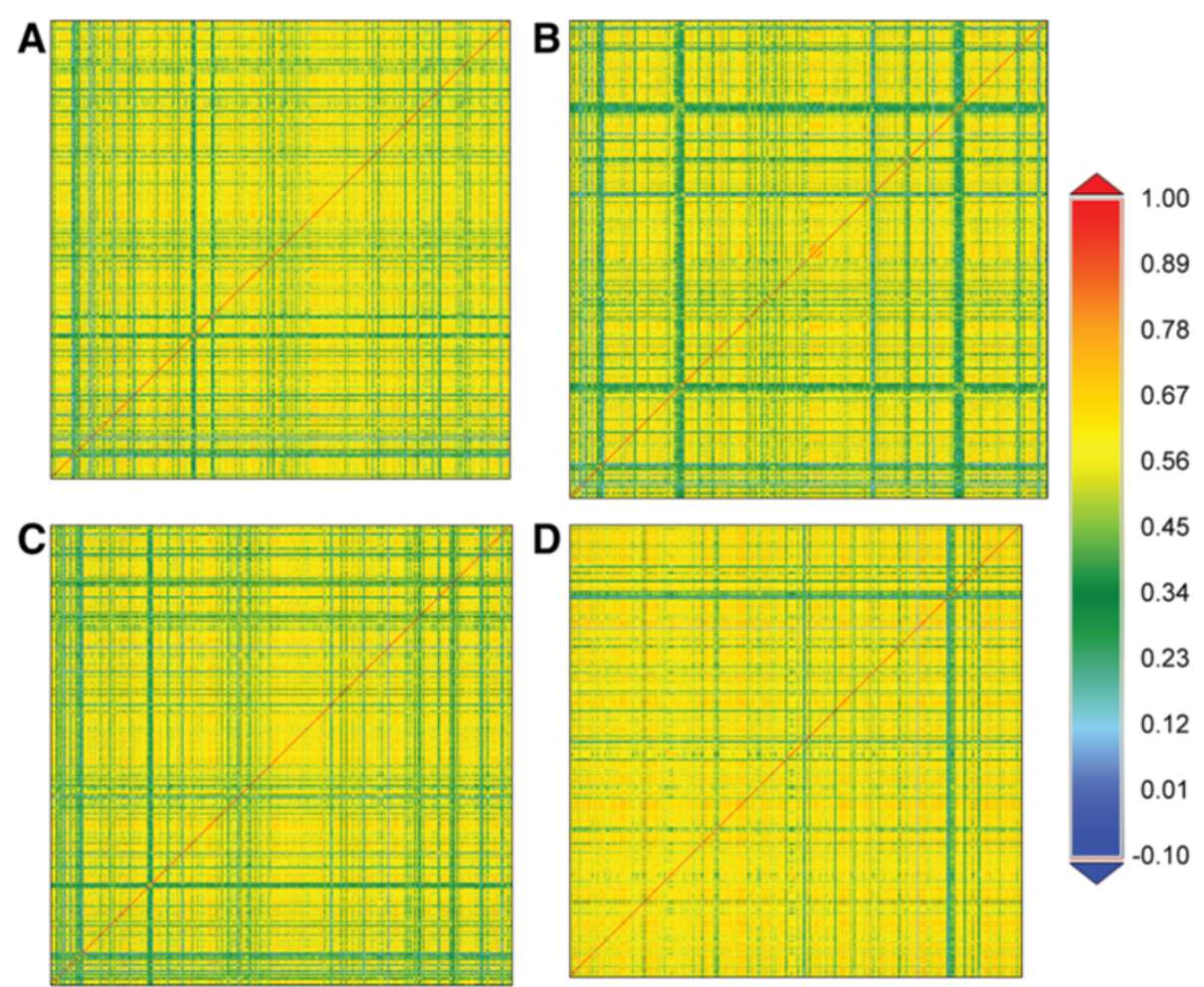

Figure 1 Four Neisseria genomes compared against themselves via S-plot. The color-coded scale on the right indicates the level of similarity. (A) serogroup A N. meningitidis Z2491 (B) serogroup B N. meningitidis MC58, (C) serogroup C N. meningitidis FAM18, and (D) N. gonorrhoeae FA 1090. 
identified as having unusual compositional properties; analysis of the location of these genes revealed that there were several clusters of adjacent genes. While some of the gRUCPs include genes which have been assigned a particular gene name and/or function, the majority (77\%) are annotated as hypothetical proteins. The complete listing of the wRUCPs and gRUCPs can be found in Additional file 6: Table S1 and Additional file 7: Table S2, respectively. Within the 414 windows identified, 715 (32\%) of the genes within these windows were also identified by the gene-by-gene analysis, indicative of larger acquisitions of gene clusters within the acquired DNA fragments. Thus, the gene-by-gene analysis provides a finer granularity for detecting regions of interest.

\section{Identifying gRUCPs shared within the Neisseria spp}

For each gRUCP identified from the gene-by-gene analysis, its frequency profiles of $k$-mers $(k=3)$ were compared to the profiles of all genes within each of the other Neisseria strains. (See Methods for a detailed description of $k$ selection.) The maximum $R^{2}$ was identified for each as a means of quickly recognizing homologous genes. We considered $R^{2}>0.9$ as likely homologies. As an example, Additional file 8: Figure S6 shows the similarities observed for the gRUCPs within the serogroup A N. meningitidis strain Z2491 and the other 17 Neisseria genomes. Figure 2 provides a summary of this analysis. Twenty-six percent (564) of the 2,172 gRUCPs identified were common amongst all 18 strains examined. (Note, while some homologous genes were classified as gRUCPs for all 18 strains, others may not have if they did not meet the required similarity threshold.) The percentage of each strain's gRUCPs that are present in all of the Neisseria strains examined varied (Figure 2B). The genes within the non-pathogenic $N$. lactamica genome classified as gRUCPs were frequently found within other Neisseria strains; roughly $35 \%$ of the gRUCPs had $R^{2}>0.9$ (Figure $2 \mathrm{~B}$ ) and $65 \%$ and $79 \%$ of the $N$. lactamica gRUCPs had an $R^{2}$ value greater than 0.8 and 0.7 , respectively. This is consistent with the notion that a non-pathogenic Neisseria species was the ancestor of all extant Neisseria species. Given the non-pathogenic nature of $N$. lactamica, genes identified as having unusual compositional properties may be GEIs or genes under different selective pressures and thus exhibiting a composition variant from the norm. In contrast the $N$. meningitidis MC58 (NC_003112) and N. gonorrhoeae NCCP11945 (NC_011035) appear to have rather unique gRUCPs; none and only two, respectively, of their gRUCPs are found within all of the other Neisseria strains (Figure 2B). While some of the genes in the 18 genomes have been annotated according to their function, many are hypothetical. Additional file 9: Table S3 lists the maximum $R^{2}$ for each gRUCP by strain.

Further investigation of the gRUCPs revealed several instances in which RUCPs were found in some but not all strains of the same species. Nevertheless, there were 57 gRUCPs which were conserved amongst all strains of pathogenic Neisseria spp., 52 gRUCPs which were conserved amongst only the $N$. meningitidis strains, and 38 gRUCPs which were conserved amongst only the $N$. gonorrhoeae strains. Given the expectation that a homologous gene could be identified as a gRUCP in more than one genome, the sequences for each of the aforementioned gRUCPs were aligned in order to identify the number of unique genes detected as having unusual compositional properties. Each of the homologous gene sequences were then compared to all publicly available bacterial nucleotide and protein sequences using BLASTn and BLASTx. Table 2 summarizes the results of this analysis. On several occasions homologies were found with other Neisseria species and strains for which complete genomic sequences are not available. For instance, half of the genes found to be conserved amongst all of the pathogenic Neisseria strains and absent from the single $N$. lactamica strain examined here (strain ST-640) BLASTed to the non-pathogenic $N$.
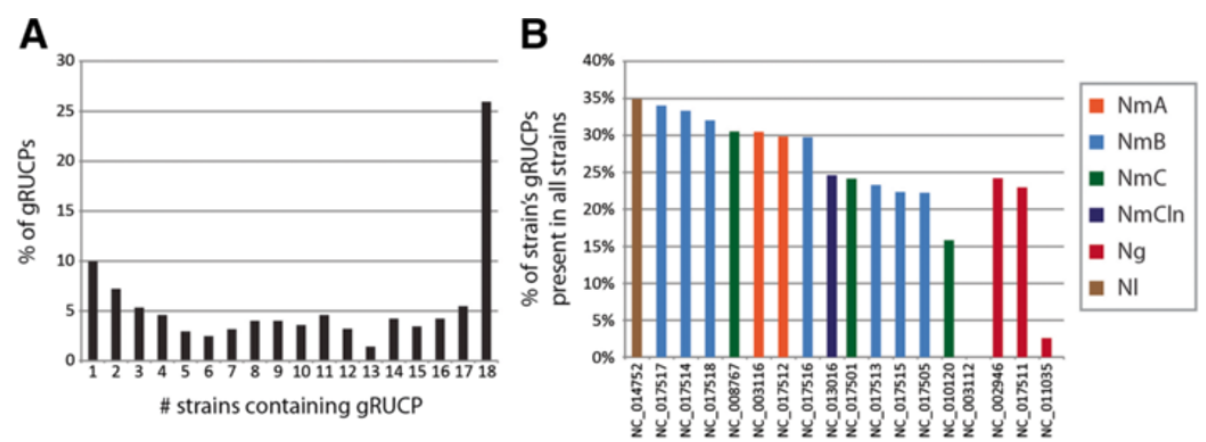

Figure 2 Examining gRUCPs common between different strains and species of Neisseria. (A) Percentage of gRUCPs identified by the gene-by-gene analysis that are unique to the strain, 1, to present in all strains examined, 18. (B) Percentage of each strain's gRUCPs that are present in all of the Neisseria strains examined. 
Table 2 Summary of homologous genes conserved amongst and within only the pathogenic Neisseria strains, the N. meningitidis strains, and the $N$. gonorrhoeae strains

\begin{tabular}{|c|c|c|}
\hline Conserved amongst & \# Homologous genes & Genes found in other genera \\
\hline \multirow[t]{2}{*}{ Pathogenic Neisseria strains } & 8 & - Putative phage associated protein present in a few Burkholderia spp. \\
\hline & & $\begin{array}{l}\text { - Annotated in other species, e.g. Moraxella catarrhalis and Haemophilus influenzae, } \\
\text { as ribonuclease T (rnt) }\end{array}$ \\
\hline \multirow[t]{6}{*}{ N. meningitidis strains } & 11 & - Putative heavy metal transport protein (NMAA_1445) \\
\hline & & - PEMK-like protein \\
\hline & & - Superoxide dismutase* \\
\hline & & - Putative heavy metal transport protein* \\
\hline & & - Hypothetical protein* \\
\hline & & - FrpC protein* \\
\hline \multirow[t]{2}{*}{ N. gonorrhoeae strains } & 13 & - 4 phage-associate proteins \\
\hline & & - Hypothetical proteins * \\
\hline
\end{tabular}

*Also found in other Neisseria species for which complete annotated genomes are not available.

lactamica strain 020-06 (whose genome is not yet complete). The similarity between the $N$. meningitidis gRUCP (NMC1806) with annotated FrpC proteins was of interest; this gene has previously been annotated as a PAI [7] and has also been explored as playing a role in meningococcal infections [44,45]. Additional file 10: Table S4, Additional file 11: Table S5, Additional file 12: Table S6, and Additional file 13: Table S7 provide details about the results of the BLAST searches for each of the genes examined.

Numerous gRUCPs were present in many but not all of the genomes for a species, indicating strain-specific losses. This was most clearly seen within the N. meningitidis sequences studied. The $N$. meningitidis strains contained 88 gRUCPs that were present within the $N$. lactamica genome and absent from the three $N$. gonorrhoeae genomes. In contrast, there were only four gRUCPs present within the $N$. gonorrhoeae genomes and the $N$. lactamica genome that were not present in any of the $N$. meningitidis strains. Under the premise that all three of these species derived from the same ancestor $[46,47]$, the presence/absence of $N$. meningitidis and $N$. gonorrhoeae gRUCPs in $N$. lactamica presents insight into their evolutionary history. Either $N$. gonorrhoeae lost the gRUCPs present within the $N$. meningitidis strains or $N$. meningitidis acquired these gRUCPs from $N$. lactamica post-speciation. As previous research has found that there is some genetic exchange between $N$. lactamica and $N$. meningitidis $[48,49]$, the former - the loss of gRUCPs by N. gonorrhoeae - is more plausible than the latter.

\section{PAI presence in RUCPs}

Referring to the Virulence Factor Database [50] and previous literature for detecting PAIs within the Neisseria spp. [3,7,28,51-54], the RUCPs (both wRUCPs and gRUCPs) were again examined looking for the major virulence factors annotated for Neisseria. Genes associated with adherence (primarily the type IV pili), capsule (siaB, $\operatorname{sia} C, \operatorname{sia} D, \operatorname{syn} X, \operatorname{lip} A, \operatorname{lip} B, \operatorname{ctr} A, \operatorname{ctr} B, c t r C$, and $\operatorname{ctr} D)$, as well as genes for invasion (opa) were all included in the genes classified as RUCPs. The capsule-related genes were identified as gRUCPs present within $N$. meningitidis and absent from the capsule-lacking $N$. gonorrhoeae strains. Another virulence factor listed in the VFDB is iron uptake as this is a critical function for the survival of Neisseria in the host. Genes including the transferrin-binding proteins $(t b p A$ and $t b p B)$, lactoferrin-binding proteins $(l b p A$ and $l b p B)$, and $A B C$-transporters, amongst others, were also identified as RUCPs. Table 3 lists the specific gene IDs for several of the aforementioned known virulence factors found as RUCPs in the N. meningitidis strains examined. (See Additional file 6: Table S1 and Additional file 7: Table S2 for additional gene IDs.)

Our analysis additionally recognized genes which have been associated with functionality that enables virulence. gRUCPs include the maf family of proteins which play a role in adhesion [55] as well as many of the comE gene copies which are involved in DNA uptake [56,57]. The

Table 3 Some of the genes which have been previously identified as Neisseria virulence factors (VFDB [50]) that were found as RUCPs in the $N$. meningitidis strains examined

\begin{tabular}{ll}
\hline Major virulence factors: & $\begin{array}{l}\text { Sampling of genes found as } \\
\text { RUCPs in } \boldsymbol{N} \text {. meningitidis strains: }\end{array}$ \\
\hline Adherence (LOS, Type IV pili) & NMA0424 \\
Antiphagocytosis (capsule) & NMBB_0072, NMBB_0073, \\
IgA1 Protease & NMBB_0073A \\
Invasion (Opa, Opc, Porin) & NMBB_0786 \\
& NMBNZ6533_NMC1877, \\
Iron uptake (FbpABC, HmbR, & NMBNZ0533_1791, NMO_1581 \\
HpuAB, Lbp, Tbp) & \\
\hline
\end{tabular}


RUCPs also contain numerous other proteins involved in the type IV secretion system, including the tra family of proteins and $\operatorname{ltg} X$, amongst others. T4SS genes are often identified by their homology to the transfer genes of conjugative plasmids or the Ti plasmid of Agrobacterium tumefaciens [58]. The gene atlA is classified as a RUCP; this gene has been found to play a critical role in bacterial resistance to phagocytosis and survival in the bloodstream in Streptococcus mutans [59]. Providing similar protection from phagocytosis [60], several of the RUCPs are or include genes annotated as $\mathrm{Cu}-\mathrm{Zn}$ superoxide dismutases (Additional file 6: Table $\mathrm{S} 1$ and Additional file 7: Table S2).

\section{Ascertaining the acquisition and putative sources for gRUCPs}

Of the gRUCPs found, 217 appear to be unique to a single Neisseria strain $\left(R^{2}>0.9\right)$ : 80 in $N$. gonorrhoeae strains, 99 in $N$. meningitidis strains, and 38 in the $N$. lactamica genome. The nucleotide sequence of each was then BLASTed against the $\mathrm{nr} / \mathrm{nt}$ Nucleotide database in an effort to find the putative source of these genes. Of the 217 genes, only 84 (17 N. gonorrhoeae, $60 \mathrm{~N}$. meningitidis and $7 \mathrm{~N}$. lactamica) produced significant similarities (Additional file 13: Table S7). (As a result of these BLAST searches, four were found to be annotated in other genera as 50 S ribosomal protein L36, although not in Neisseria, and thus removed from further analysis.) In total, 80 of these 213 genes identified as exhibiting unusual compositional properties and unique to a single Neisseria strain are present in the genomes of other organisms. Thus, these genes could either be acquired via horizontal gene transfer or their unusual compositional properties are the result of locus specific selective forces, also at play in other bacterial genomes. The remaining $64 \%$ of these genes do not show significant sequence similarity to any sequenced species in NCBI's nucleotide collection.

The BLAST hits were comprised of several hypothetical proteins as well as putative phage associated proteins from a wide variety of bacteria. Homologs to other bacterial species were also identified with annotations including: $\mathrm{Cu}-\mathrm{Zn}$ superoxide dismutase, excinuclease, chaperone protein DnaK, SecY subunit, zinc transporter, and cation efflux protein. Several of these hits were to other human bacterial pathogens, e.g. Escherichia coli, Helicobacter cinaedi, Salmonella enterica, Haemophilus influenzae, Bordetella pertussis, Pseudomonas aeruginosa and Rickettsia, amongst others (Additional file 13: Table S7). Moreover, many of the BLASTn hits of the $N$. meningitidis gRUCPs were to bacteria containing a capsule, including Pseudomonas spp., Kiebsiella spp., Haemophilus spp., Escherichia spp., Salmonella spp. and Bordetella spp.
In addition to revealing homologies with sequenced bacteria, two of the gRUCPs were found to share statistically significant similarity with sequences from eukaryotic species. One gRUCP (NMB1848), from the serogroup B strain N. meningitidis MC58, showed similarity to human and zebrafish sequences. Homology between NMB1848 and a portion of the PHD finger protein sequence PHF21B was detected in both eukaryotic species. The GO functionality associated with PHF21B is zinc ion binding (Additional file 13: Table S7). The N. meningitidis sequence exhibits the greatest sequence similarity with these and other eukaryotic species through a series of low-complexity repeats (5'-GAATACCTGAATC-3'). BLAST nucleotide searches did not identify any complete orthologous genes within the other Neisseria species; the 3' end of the gene (upstream of the repeat region) exhibited sequence similarity with non-coding regions in the genomes of $N$. gonorrhoeae and $N$. lactamica. When BLASTing the repetitive region alone, similarities with Bacillus thuringiensis as well as a number of eukaryotic species (e.g. grape, horse, European polecat, and bonobo) in addition to human (Figure 3) were uncovered. NMB1848 is CpG-rich, containing more $\mathrm{CpG}$ dinucleotides than are expected given the underlying GC-content of $N$. meningitidis. Another gRUCP (NGTW08_1668) resulted in BLAST hits to sequences from mammalian genomes. This hit corresponds to the L1 element. The acquisition of human DNA was recently found within some strains of $N$. gonorrhoeae [61]. In this previous study, evidence of a 685bp sequence exhibiting $98-100 \%$ homology with the human L1 element was found.

Under the assumption that acquired genes will adopt a more Neisseria-like composition, particularly with respect to third position mutations of the codon, one can hypothesize that genes exhibiting a composition most divergent from the genome norm are relatively recent acquisitions. The converse, however, is not possible to assert; one cannot assume that those exhibiting a more Neisseria-like composition are older as they may have been acquired from a species exhibiting a composition similar to Neisseria. As the analysis of the strain-specific gRUCPs shows, a wide variety of species can be the source of horizontally acquired genes. Genes such as phage associated proteins, the capsule proteins, the comE family of proteins and other virulence-associated proteins were amongst the most divergent from their respective genome's composition (Table 4; full listing in Additional file 14: Table S8). As this table shows, three of the most divergent genes were coding regions of the non-pathogenic $N$. lactamica strain; referring to the gRUCPs comparisons performed (Additional file 9: Table S3), these three (NLA_1460, NLA_8410 and NLA_13760) are in fact unique to $N$. lactamica, not occurring in any of the other Neisseria species nor producing any hits in our 

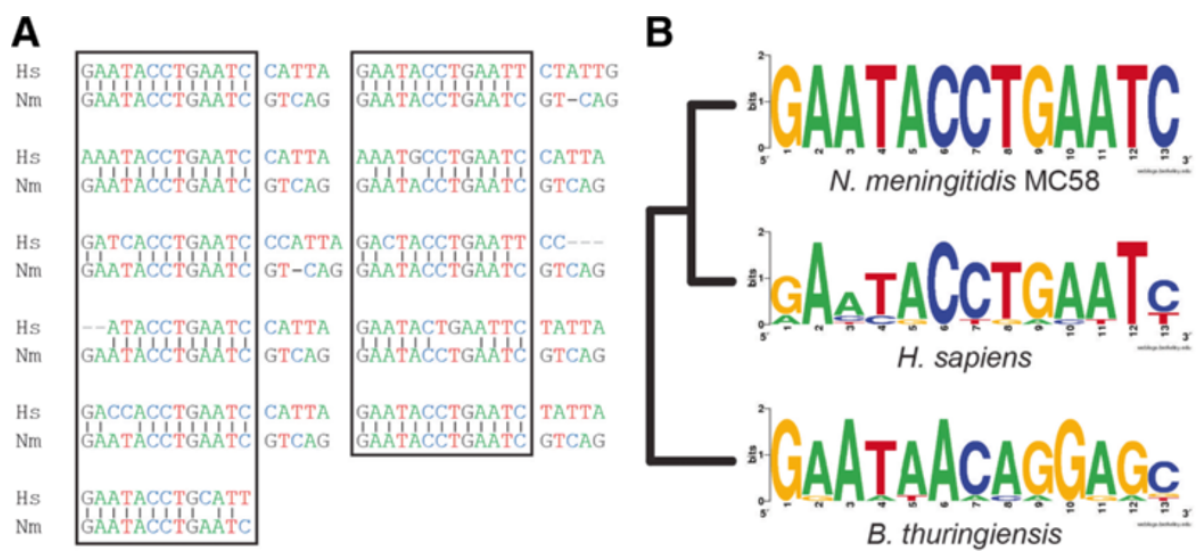

Figure 3 Repetitive sequence within NMB1848. (A) Sequence similarity between the NMB1848 sequence (Nm) and the human (Hs) sequence. The repeats found in these two sequences are included within the boxed region. (B) Sequence similarity between the consensus of the repeating sequences in N. meningitidis NMB1848, human, and B. thuringiensis. (Logos and consensus sequences created using WebLogo [62].)

BLAST search suggesting that they were acquired within the $N$. lactamica lineage relatively recently.

\section{Discussion}

From our examination of the gRUCPs found to be unique to individual Neisseria strains, a number of putative sources, including other pathogenic bacteria, were identified suggesting that gene exchange occurs between Neisseria spp. and other bacterium within their microhabitat. Furthermore, gRUCPs identified within N. meningitidis strains were homologous to a number of other bacterium containing a capsule. The homology identified between a $N$. meningitidis gRUCP and the human genome revealed for the first time that $N$. meningitidis may be capable of integrating host DNA. The majority of the gRUCPs found to be unique to individual strains, however, did not BLAST to any known species or gene. While the evolutionary path of the Neisseria genus has long been a point of debate, our examination of the gRUCPs, which includes the pathogenome of Neisseria (Table 3), suggests that $N$. meningitidis is more closely related to the non-pathogenic Neisseria species than $N$. gonorrhoeae.

This study is consistent with previous analyses of the $N$. meningitidis genomes finding the species to have an open pan-genome [34]. The approach employed here permits one to easily identify those genes most divergent to the underlying composition of the genome. Genes which are unique and likely recent acquisitions for even the non-pathogenic $N$. lactamica genome were observed signifying that the three Neisseria species are all recipients of horizontally transferred elements post-speciation. Furthermore, evidence of strain specific and serotype specific acquisitions of genes were also identified. For instance, the $N$. meningitidis MC58 and $N$. gonorrhoeae NCCP11945 genomes have a number of unique gRUCPs, suggesting that these two strains have acquired genes unique to their individual evolutionary history.

The majority of the genes which are unique to a particular strain did not reveal statistically significant homologies with any sequenced species indicating that the source of these proteins remains unknown. Because

Table 4 Top 10 genes exhibiting a composition most divergent from their genome's respective norm

\begin{tabular}{|c|c|c|c|}
\hline Gene synonym & Serogroup (Acc\#) & Protein product & $R^{2}$ Deviation \\
\hline NLA_13760 & NI (NC_014752) & Integral membrane protein & 0.4139554 \\
\hline NLA_1460 & NI (NC_014752) & Hypothetical protein & 0.4088421 \\
\hline NMO_1245 & NmCln (NC_013016) & FrpA/C-related protein, truncated & 0.4077326 \\
\hline NG00622 & $\mathrm{Ng}\left(\mathrm{NC} \_002946\right)$ & Hypothetical protein & 0.4064973 \\
\hline NMAA_0648 & NmA (NC_017512) & Hypothetical protein & 0.4000368 \\
\hline NMBM01240355_0958 & NmB (NC_017517) & Hypothetical protein & 0.39973 \\
\hline NG01938 & $\mathrm{Ng}\left(\mathrm{NC} \_002946\right)$ & Hypothetical protein & 0.3980348 \\
\hline NMAA_1127 & NmA (NC_017512) & FrpA/C-like protein & 0.3935624 \\
\hline NMA0941 & NmA (NC_003116) & Hypothetical protein & 0.3919223 \\
\hline NMO_1240 & NmCln (NC_013016) & Superoxide dismutase & 0.3910261 \\
\hline
\end{tabular}


these gRUCPs exhibited divergent nucleotide compositions, it is not likely that the genes are native to Neisseria. Rather, we hypothesize that they were acquired from another genus. For those gRUCPs which were found to be strain specific and homologous to genes within other sequenced bacterial genomes, one of two scenarios is possible: (1) the species identified from the BLAST search can be the source of the gene acquired by Neisseria or (2) both species could have acquired the gene from the same source. Looking at some of the organisms identified from the BLAST searches (Additional file 13: Table S7), one can find very probable instances of gene exchange. For instance, many of the BLAST results find similarities with sequences in Haemophilus spp. and Moraxella catarrhalis, other human pathogens.

The sequence similarity between gRUCPs and eukaryotic species was unexpected. While one of these gRUCPs has recently been discussed in the literature [61], the acquisition of human DNA was previously thought exclusive to $N$. gonorrhoeae. The BLAST result finding homology between the $N$. meningitidis gene NMB1848 and a portion of the PHD finger protein sequence PHF21B is the first report to our knowledge indicating human DNA uptake within this species. The region (195 nucleotides in length) is an annotated conserved domain, Formin Homology Region 1 characteristic of its low complexity repeats of around 12 residues [63]. This domain is not found within the genome sequences of either $N$. gonorrhoeae or N. lactamica. In fact, save a moderate homology with a sequence within the $B$. thuringiensis genome (Figure 3B), this domain appears to be eukaryotic in origin. The complete NMB1848 coding region of the MC58 strain is also present in other N. meningitidis strains, although not annotated as a coding region; therefore it was not identified as a gRUCP in the other $N$. meningitidis strains. Thus, we hypothesize that it was acquired post-speciation with $N$. gonorrhoeae and from a eukaryotic source, most likely its host. As such, the acquisition of host DNA is not exclusive to N. gonorrhoeae.

The recent sequencing of the non-pathogenic $N$. lactamica genome facilitates the identification of genes responsible for the pathogenicity of $N$. gonorrhoeae and $N$. meningitidis. Our analysis revealed two hypothetical proteins which are unique to the pathogenic Neisseria; further investigation is needed to ascertain if or how they are contributing to virulence. The gRUCPs conserved amongst all $N$. meningitidis strains and absent from $N$. gonorrhoeae and $N$. lactamica include several genes of interest, many of which are annotated as superoxide dismutase. Previous studies have found that $N$. meningitidis uses these enzymes to neutralize the effect of reactive oxygen species within the host $[64,65]$. Furthermore, in a recent transcriptomic study, these genes were found to be upregulated during infection [65]. In congruence with previous literature [66], our analysis confirms the importance of metal transport proteins for $N$. meningitidis as they are conserved amongst all of the $N$. meningitidis strains examined. The presence of the PEMK-like protein within the $N$. meningitidis genomes and absent from the $N$. gonorrhoeae genomes suggests that this may also aid in the meningococcal infection, by interfering with host mRNA [67].

The composition-based method employed here was able to identify virulence genes previously identified within the pathogenic Neisseria spp. [3,7,28,49-54]. Included are the capsule genes, some of which are present in $N$. gonorrhoeae as well as non-pathogenic species of Neisseria (Additional file 5: Figure S5). The presence of these cps-associated genes in N. gonorrhoeae, non-pathogenic Neisseria spp., as well as non-invasive meningococcal strains has led others to suggest that these genes have a biological role distinct from pathogenicity. $N$. lactamica's capsule is antigenically similar to that of $N$. meningitidis [68]. The pilin gene was identified as a gRUCP for the non-pathogenic N. lactamica. Despite its important role in pathogenicity, the presence and structure of the pilin does not indicate the species' ability to cause human disease [69]. The identification of the pilin genes as gRUCPs in the non-pathogenic as well as pathogenic Neisseriae suggests that selection is playing a role in shaping the composition and function of this gene cluster [70].

Because the vast majority of the RUCPs in $N$. lactamica are also present within the genomes of the pathogenic species, one may conclude that the two pathogenic species are more closely related to each other than either is with the non-pathogenic $N$. lactamica. This mirrors previous phylogenic analysis of the Neisseria spp. using a small set of homologous genes $[46,47]$. Unraveling the evolutionary history of the emergence of $N$. gonorrhoeae and N. meningitidis is far from trivial. Several genes were identified as acquisitions exclusive to $N$. gonorrhoeae or N. meningitidis. Moreover, genes that are present in one, two or three of the $N$. gonorrhoeae genomes and are present within all (or the majority) of the $N$. meningitidis strains examined here suggest their acquisition prior to the divergence of the two species. More baffling are the instances in which a gene is present in a single $N$. gonorrhoeae genome and in just a few (and different serotypes) of the $N$. meningitidis strains. Looking specifically at the gRUCPs present within all 18 genomes considered, a stronger similarity between $N$. meningitidis and $N$. lactamica is observed. If these gRUCPs were acquired prior to speciation of $N$. lactamica and the pathogenic species, this would suggest that $N$. meningitidis is more closely related to the non-pathogenic $N$. lactamica $[40,48,49]$. The availability of signifi- 
cantly more $N$. meningitidis genomes, however, is likely contributing to this observation. Further sequencing and annotation of $N$. gonorrhoeae genomes as well as additional Neisseria spp. is underway and will likely shed further light on the evolution of the genus.

\section{Conclusions}

The approach employed here presents a new means by which investigators can readily identify genes unique to a species or strain as well as identify genes which exhibit compositional properties aberrant from the genome norm such as acquired elements and genes under unique selective pressures. Analysis of the identified GEIs revealed that gene exchange occurs frequently between Neisseria spp. and other bacteria within their microhabitat; it also occurs with their human host. From the genic material acquired by the 18 strains examined here, albeit not a comprehensive representation of the strains present in nature, it appears that the $N$. meningitidis strains are more closely related to the non-pathogenic Neisseria lactamica than the $N$. gonorrhoeae strains are.

The genes identified as exclusive to the pathogenic Neisseria genomes, $N$. meningitidis genomes, or N. gonorrhoeae genomes provide insight into the genus both from an evolutionary perspective as well as from the perspective of that of a microbiologist. Given the prevalence of the pathogenic species, identifying additional factors which aid in the virulence of Neisseria is of importance. Genes involved in iron and zinc uptake and downstream processes have been thoroughly investigated as candidates for the development of vaccines (e.g. [71,72]). Included in the RUCPs identified here are a number of hypothetical proteins which warrant further investigation as putative virulence factors.

\section{Methods}

\section{Neisseria genome sequences}

The complete genomes of three $N$. gonorrhoeae, $14 N$. meningitidis, and one $N$. lactamica strains were obtained from NCBI's FTP site. All of these genomes have been assembled and annotated [34,36,43,54,55,73-79]. The annotation files for all these genomes were collected (".ptt and ".rnt files). Table 1 lists the genomes.

\section{Quantifying genomic similarity and dissimilarity}

The compositional profile of each window, in the case of the sliding window approach, or each gene, in the case of the gene-by-gene approach, was computed as previously described [37] and summarized as follows. Looking at an individual window or gene sequence, the frequency of occurrence of all $k$-mers is determined. When evaluating the similarity/dissimilarity of two windows or two genes, these two frequency profiles can be compared.
While a variety of different methods can be implemented, here we quantify distance according to the coefficient of determination $\left(R^{2}\right)$. The distribution $P(S)$ of appearances of all possible $k$-mers inside a given window is $P(S)=$ $N_{S} /(w-k+1)$, where $N_{S}$ and $w$ are, correspondingly, the number of appearances of $k$-mer $S$ and the total number of $k$-mers in the window being examined. The correlation statistic is appropriate only when representative statistics are collected such that $N_{s}>1$; therefore one must impose the condition $w>4^{k}$ when taking either the sliding window or gene-by-gene approach.

Analyses were conducted for a variety of different sizes of $k$ meeting the condition $w>4^{k}$. For the window-bywindow analysis with a window size of 5000 nucleotides, $k=6$ was used. wRUCPs identified for this $k$-mer size were also found when $k=5$ and $k=4$. Because the length of genes varies across the genome, the gene-by-gene analysis necessitated a choice of $k$ such that $4^{k}$ is smaller than the shortest annotated coding region. As such, the largest size $k$ which can be used is 3 . Because our analysis focuses on 3-mer and 6-mer compositional profiles (capturing the underlying codon usages), genes which do not exhibit the Neisseria codon usage and are likely from foreign sources are identified as exhibiting an unusual composition.

To assess the similarity of the compositional properties of a particular window $i$ within its genomic sequence, the average $R^{2}$ value as well as the standard deviation is calculated. Thus, windows which exhibit the same $k$-mer usage profiles will have a $R^{2}$ value approaching 1 . Windows containing regions which have not been under strong selection to follow the genome-wide composition (e.g. mono- or dinucleotide biases or codon biases), such as rRNA, will be less similar to the other windows in its genome, thus resulting in a smaller $R^{2}$ value. Likewise, foreign DNA recently integrated into the genome, e.g. horizontally transferred elements, will also be less similar to the other windows in its genome. As a threshold, we select those windows/genes having an $R^{2}$ value two standard deviations from the genome average $R^{2}$ value as exhibiting unusual compositional properties. Of the 18 genomes examined here, no windows/genes had an $R^{2}$ value greater than or equal to two standard deviations greater than the genome average. Given our interest in regions acquired from foreign sources, putative virulence factors and genes under strong selection (despite genome compositional proclivities), genes such as rRNA were not classified as RUCPs. We refer to the regions exhibiting unusual compositional properties from the window-by-window analysis as wRUCPs and from the gene-by-gene analysis gRUCPs.

The source code, including fixed and variable window functionality implemented in $\mathrm{C}++_{+}$, is freely available upon request. 


\section{Visualizing similarity and dissimilarity between genomes}

To visualize the similarity, we plot the matrix of correlation coefficients $(r), C(i, j)$, between the distributions of $k$-mers, where $i$ is a window in the first genome and $j$ is a window in the second genome. (Note, visualization is performed using $r$ values not $R^{2}$ values in an effort to expand the spectrum of values observed). The vertical and horizontal coordinates in an S-plot represent the location of windows $i$ and $j$, respectively. Different correlation coefficients are represented on the plots by different colors. An application to generate S-Plots using the C\# language for Windows was created and is freely available at www.bioinfo.uh.edu/splot. For further details, the reader is referred to [37] and the online documentation accompanying the application. The gene-by-gene functionality is not included within the visualization tool.

\section{Comparative analysis of RUCPs}

wRUCPs and gRUCPs were firstly compared to the other members of the Neisseria spp. as well as all publicly available genomes. Similarities between Neisseria spp. were conducted by examining correlations between each individual RUCP and all windows in the other Neisseria sequences. Sequences were either aligned using BioEdit (http://www.mbio.ncsu.edu/bioedit/bioedit.html) or BLASTed. BLAST-based analysis was performed as follows. For each RUCP, be it a window or an individual gene, the sequence was parsed from the complete genome. Using the BLAST web interface, each sequence was compared to all sequences within the nucleotide collection $\mathrm{nr} / \mathrm{nt}$ database using BLASTn with a word size of 7. In addition, the search was conducted with the exclusion of the taxonomical group for Neisseria (taxid: 482) and an e-value threshold of 0.5. Upon inspection of the results for each sequence, only those hits meeting the aforementioned threshold and resulting in a query coverage greater than $50 \%$ were considered homologous. Genes which uniquely appeared in a single Neisseria strain were also compared to all publicly available genomes in the nucleotide collection $\mathrm{nr} / \mathrm{nt}$ database. The same threshold, e-value and exclusion as our previous BLAST analysis were applied again here. BLASTx searches were also conducted to examine the similarity of gRUCPs conserved amongst all pathogenic Neisseria spp., amongst all $N$. meningitidis spp., and amongst all $N$. gonorrhoeae spp. with other Neisseria sequences which were not included in this analysis as well as other bacterial genomes. A word size of 2 was used for more sensitive searches. All e-values less than 1.0 were considered putative hits.

\section{Additional files}

Additional file 1: Figure S1. The S-plot of N. meningitidis MC58 (Serogroup B) vs. N. meningitidis Z2491 (Serogroup A).
Additional file 2: Figure S2. The S-plot of N. meningitidis Z2491 (Serogroup A) vs. N. gonorrhoeae FA 1090.

Additional file 3: Figure S3. The S-plot of N. meningitidis Z2491 (Serogroup A) vs. N. lactamica ST-640

Additional file 4: Figure S4. The S-plot of N. gonorrhoeae FA 1090 vs. N. lactamica ST-640.

Additional file 5: Figure S5. Compares the $N$. meningitidis serogroup $C$ strain FAM18 with (a) an N. meningitidis serogroup A strain, (b) an N. gonorrhoeae strain, and (c) the N. lactamica strain, highlighting the location of genes associated with the capsule.

Additional file 6: Table S1. Includes the complete listing of the WRUCPs identified in the 18 Neisseria strains.

Additional file 7: Table S2. Includes the complete listing of the gRUCPs identified in the 18 Neisseria strains.

Additional file 8: Figure S6. Illustrates the $R^{2}$ value for each gRUCPS identified in the serogroup A N. meningitidis Z2491 strain and the closest coding region found within in each of the other 17 genomes.

Additional file 9: Table S3. Lists the maximum homology score $\left(R^{2}\right)$ to genes within other Neisseria strains for each gRUCP.

Additional file 10: Table S4. The list of genes identified as gRUCPS which were conserved amongst all pathogenic Neisseria strains and absent from the N. lactamica ST-640 genome sequence. Carrier strains were excluded from analysis.

Additional file 11: Table S5. Lists the genes identified as gRUCPS which were conserved amongst all N. meningitidis strains but are not present in any of the examined $\mathrm{N}$. gonorrhoeae strains or the N. lactamica ST-640 genome sequence. Carrier strains were excluded from analysis.

Additional file 12: Table S6. The list of genes identified as gRUCPS which were conserved amongst all N. gonorrhoeae strains and not present in any of the examined $N$. meningitidis strains or the $N$. lactamica ST-640 genome sequence. Carrier strains were excluded from analysis.

Additional file 13: Table S7. Presents the BLASTn results for the 217 genes which appear to be unique to a single Neisseria strain.

Additional file 14: Table S8. Provides information regarding the genes exhibiting a composition most divergent from the genome norm.

Competing interests

The authors declare that they have no competing interests.

\section{Authors' contributions}

$C P, B N, S N$, and YF designed the study. CP and YF developed the software. MS and CP carried out the bioinformatic analysis. All authors participated in the drafting of the manuscript as well as read and approved the final manuscript.

\section{Acknowledgments}

The authors would like to thank Audrey Hart-van Tassell, Petri Urvil, Meizhuo Zhang, Sergei Chumakov for their contributions in the initial efforts in this project. MS was partially supported through Loyola University Chicago's Biology Summer Research Fellowship.

\section{Author details}

'Department of Biology, Loyola University Chicago, 1032 W. Sheridan Rd, Chicago, IL 60660, USA. ²Department of Computer Science, Loyola University Chicago, Chicago, IL 60660, USA. ${ }^{3}$ Bioinformatics Program, Loyola University Chicago, Chicago, IL 60626, USA. ${ }^{4}$ Department of Obstetrics \& Gynecology, Meharry Medical College, Nashville, TN 37208, USA. ${ }^{5}$ Department of Computer Science, University of Houston, Houston, TX 77204, USA.

${ }^{6}$ Department of Biology and Biochemistry, University of Houston, Houston, TX 77204, USA. 


\section{References}

1. Lee CA: Pathogenicity islands and evolution of bacterial pathogens. Infect Agent Dis 1996, 5:1-7

2. Hacker J, Blum-Oehler G, Muhldorfer I, Tschape H: Pathogenicity islands of virulent bacteria: structure, function and impact on microbial evolution. Mol Microbiol 1997, 23:1089-1097.

3. Dillard JP, Seifert HS: A variable genetic island specific for Neisseria gonorrhoeae is involved in providing DNA for natural transformation and is found more often in disseminated infection isolates. Mol Microbiol 2001, 41:263-277.

4. Hentschel U, Hacker J: Pathogenicity islands: the tip of the iceberg Microbes Infect 2001, 3:545-548.

5. Lawrence JG, Ochman H: Amelioration of bacterial genomes: rates of change and exchange. J Mol Evol 1997, 44:383-397.

6. Lawrence JG, Roth JR: Roles of horizontal transfer in bacterial evolution. In Horizontal Transfer. Edited by Syvanen M, Kado Cl. London: Chapman and Hall; 1998:208-225.

7. Lio $P$, Vannucci M: Finding pathogenicity islands and gene transfer events in genome data. Bioinformatics 2000, 16:932-940.

8. Ochman H, Lawrence JG: Phylogenetics and the amelioration of bacterial genomes. In Escherichia coli and Salmonella typhimurium: Cellular and Molecular Biology. Edited by Neidhardt FC, Curtiss R III, Ingraham JL, Lin ECC, Low KB. Washington, DC: American Society for Microbiology; 1996:2627-2637.

9. Sueoka N: Two aspects of DNA base composition: $\mathrm{G}+\mathrm{C}$ content and translation-coupled deviation from intra-strand rule of $A=T$ and $G=C$. J Mol Evol 1999, 49:49-62.

10. Karlin S, Mrazek J: Predicted highly expressed genes of diverse prokaryotic genomes. J Bacterio/ 2000, 182:5238-5250.

11. Karlin S, Mrazek J, Campbell AM: Codon usages in different gene classes of the Escherichia coli genome. Mol Microbiol 1998, 29:1341-1355.

12. Medigue C, Rouxel T, Vigier P, Henaut A, Danchin A: Evidence for horizontal gene transfer in Escherichia coli speciation. J Mol Biol 1991, 222:851-856.

13. Moszer I, Rocha EP, Danchin A: Codon usage and lateral gene transfer in Bacillus subtilis. Curr Opin Microbiol 1999, 2:524-528.

14. Sharp PM, Li W-H: The codon adaptation index - a measure of directional synonymous codon usage bias, and its potential applications. Nucl Acids Res 1987, 15:1281-1295.

15. Whittam TS, Ake S: Genetic polymorphisms and recombination in natural populations of Escherichia coli. In Mechanisms of Molecular Evolution. Edited by Takahata N, Clark AG. Tokyo: Japan Scientific Society Press; 1993:223-246.

16. Hooper SD, Berg OG: Detection of genes with atypical nucleotide sequence in microbial genomes. J Mol Evol 2002, 54:365-375.

17. Karlin S, Burge C: Dinucleotide relative abundance extremes: a genomic signature. Trends Gent 1995, 11:283-290.

18. Worning $P$, Jensen $L$, Nelson KE, Brunak S, Ussery DW: Structural analysis of DNA sequence: evidence for lateral gene transfer in Thermotoga maritima. Nucl Acids Res 2000, 28:706-709.

19. Ventura M, Canchaya C, vanSinderen D, Fitzgerald GF, Zink R: Bifidobacterium lactis DSM 10140: Identification of the atp (atpBEFHAGDC) operon and analysis of its genetic structure, characteristic, and phylogeny. App/ Environ Microbiol 2004, 70:3110-3121.

20. Hayes WS, Borodovsky M: How to interpret an anonymous bacterial genome: machine learning approach to gene identification. Genome Res 1998, 8:1154-1171.

21. Aravind L, Tatusov RL, Wolf Yl, Walker DR, Koonin EV: Evidence for massive gene exchange between archaeal and bacterial hyperthermophiles. Trends Genet 1998, 14:442-444.

22. Doolittle RF, Feng DF, Anderson KL, Alberro MR: A naturally occurring horizontal gene transfer from a eukaryote to a prokaryote. J Mol Evol 1990, 31:383-388.

23. Lawrence JG, Hartl DL: Inference of horizontal genetic transfer from molecular date: an approach using the bootstrap. Genetics 1992, 131:753-760.

24. Logsdon JM, Furguy DM: Thermotoga heats up lateral gene transfer. Curr Biol 1999, 9:R747-R751.

25. Nelson KE, Clayton RA, Gill SR, Gwinn ML, Dodson RJ, Haft DH, Hickey EK, Peterson JD, Nelson WC, Ketchum KA, McDonald L, Utterback TR, Malek JA Linher KD, Garrett MM, Steward AM, Cotton MD, Pratt MS, Phillips CA, Richardson D, Heidelberg J, Sutton GG, Fleischmann RD, Eisen JA, White O, Salzberg SL, Smith HO, Venter JC, Fraser CM: Evidence for lateral gene transfer between Archaea and bacteria from genome sequence of Thermotoga maritima. Nature 1999, 399:323-329.

26. Ragan MA: On surrogate methods for detecting lateral gene transfer. FEMS Microbiol Lett 2001, 201:187-191.

27. Ragan MA: Detection of lateral gene transfer among microbial genomes. Curr Opin Genet Dev 2001, 11:620-626.

28. Karlin S: Detecting anomalous gene clusters and pathogenicity islands in diverse bacterial genomes. Trends Microbiol 2001, 9:335-343.

29. Garcia-Vallvé S, Romeu A, Palau J: Horizontal gene transfer in bacterial and archaeal complete genomes. Genome Res 2000, 10:1719-1725.

30. Lawrence JG, Ochman H: Molecular archeology of the Escherichia coli genome. Proc Natl Acad Sci USA 1998, 95:9413-9417.

31. Ochman H, Lawrence JG, Groisman E: Lateral gene transfer and the nature of bacterial innovation. Nature 2000, 405:299-304.

32. The Gonococcal Genome Sequencing Project. http://www.genome.ou.edu/ gono.html.

33. Zhang R, Zhang C-T: A systematic method to identify genomic islands and its applications in analyzing the genomes of Corynebacterium glutamicum and Vibrio vulnificus CMCP6 chromosome 1. Bioinformatics 2004, 20:612-622.

34. Schoen C, Blom J, Claus H, Schramm-Glück A, Brandt P, Müller T, Goesmann A, Joseph B, Konietzny S, Kurzai O, Schmitt C, Friedrich T, Linke B, Vogel U, Frosch M: Whole-genome comparison of disease and carriage strains provides insights into virulence evolution in Neisseria meningitidis. Proc Natl Acad Sci USA 2008, 105:3473-3478.

35. Kislyuk AO, Katz LS, Agrawal S, Hagen MS, Conley AB, Jayaraman P, Nelakuditi V, Humphrey JC, Sammons SA, Govil D, Mair RD, Tatti KM, Tondella ML, Harcourt BH, Mayer LW, Jordan IK: A computational genomics pipeline for prokaryotic sequencing projects. Bioinformatics 2010, 26:1819-1826.

36. Budroni S, Siena E, Hotopp JCD, Seib KL, Serruto D, Nofroni C, Comanducci M, Riley DR, Daugherty SC, Angiuoli SV, Covacci A, Pizza M, Rappuoli R, Moxon ER, Tettelin H, Medini D: Neisseria meningitidis is structured in clades associated with restriction modification systems that modulate homologous recombination. Proc Natl Acad Sci USA 2011, 108:4494-4499.

37. Putonti C, Luo Y, Katili C, Chumakov S, Fox GE, Willson RC, Fofanov Y: A computational tool for the genomic identification of regions of unusual compositional properties and its utilization in the detection of horizontally transferred sequences. Mol Biol Evol 2006, 23:1863-1868.

38. Dos-Reis M, Savva R, Wernisch L: Solving the riddle of codon usage preferences: a test for translational selection. Nucleic Acids Res 2004, 32:5036-5044

39. Sharp PM, Bailes E, Grocock RJ, Peden JF, Sockett RE: Variation in the strength of selected codon usage bias among bacteria. Nucleic Acids Res 2005, 33:1141-1153.

40. Vázquez JA, de la-Fuente L, Berron S, O'Rourke M, Smith NH, Zhou J, Spratt BG: Ecological separation and genetic isolation of Neisseria gonorrhoeae and Neisseria meningitidis. Curr Biol 1993, 3:567-572.

41. Frosch M, Müller A: Phospholipid substitution of capsular polysaccharides and mechanisms of capsule formation in Neisseria meningitidis. Mol Microbiol 1993, 8:483-493.

42. Claus H, Maiden MCJ, Maag R, Frosch M, Vogel U: Many carried meningococci lack the genes required for capsule synthesis and transport. Microbiology 2002, 148:1813-1819.

43. Schoen C, Weber-Lehmann J, Blom J, Joseph B, Goesmann A, Strittmatter A, Frosch M: Whole-genome sequence of the transformable Neisseria meningitidis serogroup A strain WUE2594. J Bacteriol 2011, 193:2064-2065.

44. Osička R, Kalmusová J, Kř́žová P, Šebo P: Neisseria meningitidis RTX protein FrpC induces high levels of serum antibodies during invasive disease: polymorphism of frpC alleles and purification of recombinant FrpC. Infect Immun 2001, 69:5509-5519.

45. Forman S, Linhartova I, Osička R, Nassif X, Šebo P, Pelicic V: Neisseria meningitidis RTX proteins are not required for virulence in infant rats. Infect Immun 2003, 71:2253-2257.

46. Bennett JS, Bentley SD, Vernikos GS, Quail MA, Cherevach I, White B, Parkhill J, Maiden MC: Independent evolution of the core and accessory gene sets in the genus Neisseria: insights gained from the genome of Neisseria lactamica isolate 020-06. BMC Genomics 2010, 11:652.

47. Bennett JS, Jolley KA, Earle SG, Corton C, Bentley SD, Parkhill J, Maiden MC: A genomic approach to bacterial taxonomy: an examination and proposed reclassification of species within the genus Neisseria. Microbiology 2012, 158:1570-1580. 
48. Bennett JS, Jolley KA, Sparling PF, Saunders NJ, Hart CA, Feavers IM, Maiden MC: Species status of Neisseria gonorrhoeae: evolutionary and epidemiological inferences from multilocus sequence typing. BMC Biol 2007, 5:35.

49. Hanage WP, Fraser C, Spratt BG: Fuzzy species among recombinogenic bacteria. BMC Biol 2005, 3:6.

50. Chen $L H$, Xiong ZH, Sun LL, Yang J, Jin Q: VFDB 2012 update: toward the genetic diversity and molecular evolution of bacterial virulence factors. Nucleic Acids Res 2012, 40:D641-D645.

51. Zhu P, Morelli G, Achtman M: The opcA and (psi)opcB regions in Neisseria: genes, pseudogenes, deletions, insertion elements and DNA islands. Mol Microbiol 1999, 33:635-650.

52. Tinsley CR, Nassif $X$ : Analysis of the genetic differences between Neisseria meningitidis and Neisseria gonorrhoeae: two closely related bacteria expressing two different pathogenicities. Proc Natl Acad Sci USA 1996, 93:11109-11114.

53. Klee SR, Nassif X, Kusecek B, Merker P, Beretti JL, Achtman M, Tinsley CR: Molecular and biological analysis of eight genetic islands that distinguish Neisseria meningitidis from the closely related pathogen Neisseria gonorrhoeae. Infect Immun 2000, 68:2082-2095.

54. Tettelin $H$, Saunders NJ, Heidelberg J, Jeffries AC, Nelson KE, Eisen JA, Ketchum KA, Hood DW, Peden JF, Dodson RJ, Nelson WC, Gwinn ML, DeBoy R, Peterson JD, Hickey EK, Haft DH, Salzberg SL, White O, Fleischmann RD, Dougherty BA, Mason T, Ciecko A, Parksey DS, Blair E, Cittone $\mathrm{H}$, Clark EB, Cotton MD, Utterback TR, Khouri H, Qin H, et al: Complete genome sequence of Neisseria meningitidis serogroup B strain MC58. Science 2000, 287:1809-1815.

55. Parkhill J, Achtman M, James KD, Bentley SD, Churcher C, Klee SR, Morelli G, Basham D, Brown D, Chillingworth T, Davies RM, Davis P, Devlin K, Feltwell T, Hamlin N, Holroyd S, Jagels K, Leather S, Moule S, Mungall K, Quail MA Rajandream MA, Rutherford KM, Simmonds M, Skelton J, Whitehead S, Spratt BG, Barrell BG: Complete DNA sequence of serogroup A strain of Neisseria meningitidis Z2491. Nature 2000, 404:502-505.

56. Chen I, Gotschlich EC: ComE, a competence protein from Neisseria gonorrhoeae with DNA-binding activity. J Bacterio/ 2001, 183:3160-3168.

57. Smith MG, Gianoulis TA, Pukatzski S, Mekalanos JJ, Ornston LN, Gerstein M, Snyder M: New insights into Acinetobacter baumannii pathogenesis revealed by high-density pyrosequencing and transposon mutagenesis. Genes Dev 2007, 21:601-614.

58. Christie PJ: Type IV secretion: intercellular transfer of macromolecules by systems ancestrally related to conjugation machines. Mol Microbiol 2001, 40:294-305.

59. Jung CJ, Zheng QH, Shieh YH, Lin CS, Chia JS: Streptococcus mutans autolysin AtIA is a fibronectin-binding protein and contributes to bacterial survival in the bloodstream and virulence for infective endocarditis. Mol Microbiol 2009, 74:888-902.

60. Dunn KL, Farrant JL, Langford PR, Kroll JS: Bacterial [Cu, Zn]-cofactored superoxide dismutase protects opsonized, encapsulated Neisseria meningitidis from phagocytosis by human monocytes/macrophages. Infect Immun 2003, 71:1604-1607.

61. Anderson MT, Seifert HS: Opportunity and means: horizontal gene transfer from the human host to a bacterial pathogen. MBio 2011, 2:e00005-e00011.

62. Crooks GE, Hon G, Chandonia JM, Brenner SE: WebLogo: A sequence logo generator. Genome Res 2004, 14:1188-1190

63. Marchler-Bauer A, Zheng C, Chitsaz F, Derbyshire MK, Geer LY, Geer RC, Gonzales NR, Gwadz M, Hurwitz DI, Lanczycki CJ, Lu F, Lu S, Marchler GH, Song JS, Thanki N, Yamashita RA, Zhang D, Bryant SH: CDD: conserved domains and protein three-dimensional structure. Nucleic Acids Res 2013, 41:D348-D352.

64. Seib KL, Tseng HJ, McEwan AG, Apicella MA, Jennings MP: Defenses against oxidative stress in Neisseria gonorrhoeae and Neisseria meningitidis: distinctive systems for different lifestyles. J Infect Dis 2004, 190:136-147.

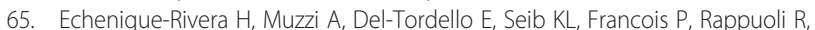
Pizza M, Serruto D: Transcriptome analysis of Neisseria meningitidis in human whole blood and mutagenesis studies identify virulence factors involved in blood survival. PLoS Pathog 2011, 7:e1002027.

66. Grifantini R, Sebastian S, Frigimelica E, Draghi M, Bartolini E, Muzzi A, Rappuoli R, Grandi G, Genco CA: Identification of iron-activated and repressed Fur-dependent genes by transcriptome analysis of Neisseria meningitidis group B. Proc Natl Acad Sci USA 2003, 100:9542-9547.
67. Zhang J, Zhang Y, Zhu L, Suzuki M, Inouye M: Interference of mRNA function by sequence-specific endoribonuclease PemK. J Biol Chem 2004, 279:20678-20684.

68. Martin PV, Laviotola A, Ohayon H, Riou JY: Presence of a capsule in Neisseria lactamica, antigenically similar to the capsule of $N$. meningitidis. Ann Inst Pasteur Microbiol 1986, 137A:279-285.

69. Aho EL, Keating AM, McGillivray SM: A comparative analysis of pilin genes from pathogenic and non-pathogenic Neisseria species. Microb Pathog 2000, 38:81-88.

70. Cahoon LA, Seifert HS: An alternative DNA structure is necessary for pilin antigenic variation in Neisseria gonorrhoeae. Science 2009, 325:764-767.

71. Pettersson A, Kortekaas J, Weynants VE, Voet P, Poolman JT, Bos MP, Tommassen J: Vaccine potential of the Neisseria meningitidis lactoferrinbinding proteins LbpA and LbpB. Vaccine 2006, 24:3545-3557.

72. Stork M, Bos MP, Jongerius I, De-Kok N, Schilders I, Weynants VE, Poolman JT, Tommassen J: An outer membrane receptor of Neisseria meningitidis involved in zinc acquisition with vaccine potential. PLoS Pathog 2010, 6:1000969.

73. Piet JR, Veld RAH, Van-Schaik BD, Van-Kampen AH, Baas F, Van De-Beek D, Pannekoek Y, Van Der-Ende A: Genome sequence of Neisseria meningitidis serogroup B strain H44/76. J Bacteriol 2011, 193:2371-2372.

74. Joseph B, Schneiker-Bekel S, Schramm-Glück A, Blom J, Claus H, Linke B, Schwarz RF, Becker A, Goesmann A, Frosch M, Schoen C: Comparative genome biology of a serogroup $B$ carriage and disease strain supports a polygenic nature of meningococcal virulence. J Bacteriol 2010, 192:5363-5377.

75. Rusniok C, Vallenet D, Floquet S, Ewles H, Mouzé-Soulama C, Brown D, Lajus A, Buchrieser C, Médigue C, Glaser P, Pelicic V: NeMeSys: a biological resource for narrowing the gap between sequence and function in the human pathogen Neisseria meningitidis. Genome Biol 2009, 10:R110.

76. Peng J, Yang L, Yang F, Yang J, Yan Y, Nie H, Zhang X, Xiong Z, Jiang $Y$, Cheng F, Xu X, Chen S, Sun L, Li W, Shen Y, Shao Z, Liang X, XU J, Jin Q: Characterization of ST-4821 complex, a unique Neisseria meningitidis clone. Genomics 2008, 91:78-87.

77. Bentley SD, Vernikos GS, Snyder LA, Churcher C, Arrowsmith C, Chillingworth T, Cronin A, Davis PH, Holroyd NE, Jagels K, Maddison M, Moule S, Rabbinowitsch E, Sharp S, Unwin L, Whitehead S, Quail MA, Achtman M, Barrell B, Saunders NJ, Parkhill J: Meningococcal genetic variation mechanisms viewed through comparative analysis of serogroup C strain FAM18. PLoS Genet 2007, 3:e23.

78. Chung GT, Yoo JS, Oh HB, Lee YS, Cha SH, Kim SJ, Yoo CK: Complete genome sequence of Neisseria gonorrhoeae NCCP11945. J Bacteriol 2008, 190:6035-6036.

79. Chen CC, Hsia KC, Huang CT, Wong WW, Yen MY, Li LH, Lin KY, Chen KW, Li SY: Draft genome sequence of a dominant, multidrug-resistant Neisseria gonorrhoeae strain, TCDC-NG08107, from a sexual group at high risk of acquiring human immunodeficiency virus infection and syphilis. $J$ Bacteriol 2011, 193:1788-1789.

doi:10.1186/1471-2148-13-184

Cite this article as: Putonti et al:: Where does Neisseria acquire foreign DNA from: an examination of the source of genomic and pathogenic islands and the evolution of the Neisseria genus. BMC Evolutionary Biology 2013 13:184.

\section{Submit your next manuscript to BioMed Central and take full advantage of:}

- Convenient online submission

- Thorough peer review

- No space constraints or color figure charges

- Immediate publication on acceptance

- Inclusion in PubMed, CAS, Scopus and Google Scholar

- Research which is freely available for redistribution 